\title{
ДИНАМИКА КИНЕМАТИЧЕСКОГО ПОРТРЕТА ПОСТИНСУЛЬТНОГО ПАРЕЗА РУКИ НА ФОНЕ РЕАБИЛИТАЦИИ
}

А. Е. Хижникова $\bowtie$, А. С. Клочков, А. М. Котов-Смоленский, Н. А. Супонева, М. А. Пирадов

Научный центр неврологии, Москва, Россия

По данным литературы, только 5-20\% пациентов после инсульта могут полностью восстановить двигательную функцию руки. Важны корректная постановка целей и индивидуальный подход, направленный на восстановление функционального статуса пациента. Целью исследования было на основании клинико-биомеханического анализа разработать алгоритм оценки нарушения двигательной функции руки у пациентов после инсульта и определить принципы выбора тактики реабилитации. В исследование были включены 25 пациентов с инсультом полушарной локализации и 10 здоровых добровольцев. Для оценки двигательной функции руки применяли формализованные клинические шкалы (шкала Фугл-Мейера, Эшворта, тест АRAT) и видеоанализ движений. Пациенты были разделены на 2 группы по степени тяжести пареза руки (легкий/умеренный и грубый/выраженный). В обеих группах проводили курс реабилитации, включавший механотерапию, массаж, ЛФК. Выявлено, что у пациентов 1-й группы восстановление двигательной функции в паретичной руке происходит по пути нормализации паттерна движения: нормализация биомеханических параметров, прямо коррелирующая с уменьшением клинической выраженности степени пареза по шкале Фугл-Мейера $(r=0,94 ; p=0,01)$. У пациентов 2-й группы восстановление двигательной функции в паретичной руке происходит по пути компенсации двигательного дефицита: сохранение патологической синергии по данным биомеханического анализа, обратно коррелирующее с уменьшением клинической выраженности степени пареза $(r=-0,9 ; p=0,03)$. В результате проведенного исследования сформирован алгоритм выбора тактики ведения пациентов, основанный на исходных клинических показателях.

Ключевые слова: инсульт, парез в руке, нейрореабилитация, адаптация, двигательное переобучение, биомеханика движений, видеоанализ движений, патологическая синергия

Финансирование: работа выполнена в рамках государственного заказа № 0512-2014-0036.

Информация о вкладе авторов: А. Е. Хижникова - планирование исследования, анализ литературы, сбор, анализ и интерпретация данных, подготовка черновика рукописи; А. С. Клочков - планирование исследования, анализ литературы, интерпретация данных, подготовка рукописи; А. М. Котов-Смоленский - проведение тренировок с пациентами, включенными в исследование, осмотр пациентов по клиническим шкалам; Н. А. Супонева - планирование исследования, интерпретация данных, подготовка рукописи; М. А. Пирадов — подготовка рукописи.

Соблюдение этических стандартов: исследование одобрено этическим комитетом ФГБНУ НЦН (протокол № 1-5/16 от 27 января 2016 г.). Все пациенты подписали добровольное информированное согласие на участие в исследовании.

$\varangle$ Для корреспонденции: Анастасия Евгеньевна Хижникова

Волоколамское шоссе, д. 80, г. Москва, 125367; nastushkapal@gmail.com

Статья получена: 16.08.2019 Статья принята к печати: 30.08.2019 Опубликована онлайн: 31.08.2019

DOI: $10.24075 /$ vrgmu.2019.056

\section{DYNAMICS OF POST-STROKE HAND PARESIS KINEMATIC PATTERN DURING REHABILITATION}

Khizhnikova AE $\bowtie$, Klochkov AS, Kotov-Smolenskiy AM, Suponeva NA, Piradov MA

Research Center of Neurology, Moscow, Russia

According to the literature data, only $5-20 \%$ of post-stroke patients are able to restore the hand motor function completely. Correct goal setting and individual approach to the patient's functional recovery are important. Our study aimed to develop an algorithm of impaired hand motor functioning assessment for post-stroke patients and to determine the principles of the rehabilitation tactics choosing based on the biomechanical analysis. Twenty five patients with hemispheric stroke and 10 healthy volunteers participated in the study. Formal clinical observation scales (Fugl-Meyer Assessment, Ashworth Scale, ARAT) and video motion analysis were used for evaluation of the hand motor function. Patients were divided into 2 groups according to the hand paresis severity (mild/moderate and pronounced/severe). Rehabilitation was carried out in both groups, including mechanotherapy, massage and physical therapy. It was revealed that in the 1st group of patients the motor function recovery in the paretic hand was due to movement performance recovery: biomechanical parameters restoration directly correlated with a decrease in the paresis degree according to the Fugl-Meyer Assessment Scale $(r=0.94 ; p=0.01)$. In the 2 nd group of patients, the motor function recovery in the paretic hand was due to motor deficit compensation: according to biomechanical analysis, the pathological motor synergies inversely correlated with a decrease in the paresis degree $(r=-0.9 ; p=0.03)$. As a result of the study, an algorithm for selecting the patient management tactics based on the baseline clinical indicators was developed.

Keywords: stroke, hand paresis, neurorehabilitation, adaptation, motor relearning, movement biomechanics, motion capture, abnormal synergy

Funding: the study was performed as a part of the public contract № 0512-2014-0036.

Author contribution: Khizhnikova AE — research planning, literature analysis, data acquisition, analysis and interpretation, manuscript draft writing; Klochkov AS — research planning, literature analysis, data interpretation, manuscript writing; Kotov-Smolenskiy AM — training of surveyed patients, patients examination using clinical scales; Suponeva NA — research planning, data interpretation, manuscript writing; Piradov MA — manuscript writing.

Compliance with ethical standards: the study was approved by the Ethics Committee of Research Center of Neurology (protocol № 5/16 dated January 27, 2016). All enrolled patients signed informed consent to participation in the study.

$\square$ Correspondence should be addressed: Anastasia E. Khizhnikova Volokolamskoye Shosse 80, Moscow, 125367; nastushkapal@gmail.com

Received: 16.08.2019 Accepted: 30.08.2019 Published online: 31.08.2019

DOI: $10.24075 /$ brsmu.2019.056

По данным ряда авторов, в остром периоде инсульта парез руки можно встретить в 48-77\% случаев [1, 2]. В то же время только 5-20\% пациентов могут полностью восстановить двигательную функцию паретичной руки к концу раннего восстановительного периода [3, 4].
Восстановление двигательной функции верхней конечности проходит за шесть последовательных стадий (от вялого пареза до возможности совершать сложные координированные движения), при этом улучшение может завершиться на любом из этапов и пациент останется с 
частично или полностью утраченными возможностями самообслуживания [5]. В связи с этим важным условием эффективной двигательной реабилитации является определение тактики реабилитации пациента для достижения максимального функционального восстановления в зависимости от текущего его этапа.

Известно, что у пациентов, перенесших инсульт, имеющих выраженный парез и повышение мышечного тонуса, физиологический паттерн движений становится невозможным. Вследствие этого возникают предпосылки для развития новых двигательных синергий, являющихся по своей сути компенсаторным механизмом. В результате организм использует для совершения двигательного акта сохранившиеся двигательные функции конечности либо активные движения в смежных суставах и функционально связанных кинематических цепях. Использование в составе компенсаторных синергий движений с более низким уровнем регуляции приводит к снижению степени приспосабливаемости К изменяющимся условиям окружающей среды. Впоследствии компенсаторные синергии приобретают патологический характер [6], что ведет к снижению функциональных возможностей пациента и замедлению темпов дальнейшей реабилитации

Тем не менее стоит отметить, что, по данным некоторых авторов, механизмы компенсации необходимы для пациентов с грубым парезом и их наличие важно для успешного формирования движений у пациентов, перенесших инсульт [7]. В ходе процесса восстановления двигательные синергии проявляются более комплексно и становятся тесно связанными со спастичностью и содружественными реакциями. В настоящее время принято считать, что для лучшего функционального двигательного восстановления необходимо проводить тренировку в рамках существующего патологического стереотипа с последующим расширением зоны активных движений [8]. Благодаря этому на фоне двигательных тренировок, как правило, происходит перестройка патологической синергии за счет увеличения объема «выгодных» компонентов движения [9].

Важны корректная постановка целей и индивидуальный подход в разработке реабилитационной программы, направленной на восстановление прежде всего функционального статуса пациента. Видеоанализ движений паретичной руки и плечевого пояса с подробной оценкой межсуставных взаимоотношений и кинематических характеристик на фоне курса реабилитации может оказать неоценимую помощь в ретроспективной оценке успешности восстановительного процесса. Целью исследования было на основании клинического и биомеханического анализов разработать принципы выбора тактики реабилитации двигательной функции руки у пациентов, перенесших нарушение мозгового кровообращения.

\section{МАТЕРИАЛЫ И МЕТОДЫ}

Исследование проводили на базе отделения нейрореабилитации и физиотерапии ФГБНУ «Научный центр неврологии» (2017-2018 гг.). Критерии включения пациентов в исследование: пациенты мужского и женского пола в возрасте 18-80 лет; наличие подтвержденного нарушения мозгового кровообращения по ишемическому или геморрагическому типу; единичный очаг поражения полушарной локализации давностью от 3-х месяцев до 2-х лет; наличие постинсультного пареза в руке от 2 до 4 баллов по Британской шкале оценки мышечной силь
[10]. Критерии исключения: степень пареза в руке меньше 2 баллов по Британской шкале оценки мышечной силы; грубое нарушение глубокой чувствительности; неглектсиндром; повышение мышечного тонуса по шкале Эшворта больше 2 баллов (0 баллов соответствуют нормальному мышечному тонусу); грубое нарушение зрения, не позволяющее различать изображение на экране компьютера; выраженные когнитивные нарушения, затрудняющие выполнение инструкций; грубая сенсорная или моторная афазия; леворукость по данным Эдинбургского опросника мануальной асимметрии [11]. В исследование было включено 25 пациентов, перенесших нарушение мозгового кровообращения полушарной локализации. Среди них было 17 мужчин и 8 женщин в возрасте 30-80 лет (медиана возраста - 55 [45; 61]). Давность инсульта составила от 3 до 23 месяцев (медиана давности инсульта 7 месяцев [4; 12]). При этом 9 больных (36\%) наблюдали в раннем восстановительном периоде, 9 больных (36\%) - в позднем восстановительном периоде, 7 больных (28\%) в резидуальном. В исследование не включали пациентов с тяжелой степенью спастичности, грубыми речевыми и когнитивными нарушениями, ограничивающими возможность коммуникации и следования указаниям инструктора-методиста по лечебной гимнастике.

Для определения нормального кинематического портрета движения руки было отобрано 10 здоровых добровольцев в возрасте 24-42 года (4 женщины и 6 мужчин) с доминантной правой рукой без патологий опорно-двигательной и нервной систем. У каждого испытуемого был проведен анализ движений как в доминантной (правой), так и в недоминантной (левой) руке.

Для клинической оценки двигательного дефицита, выраженности патологических синергий, рефлекторной активности, поверхностной и глубокой чувствительности, координации, объема пассивных движений и болевых ощущений при движениях в пораженных конечностях использовали шкалу Фугл-Мейера [12]: раздел шкалы для оценки функции руки (общий максимум баллов по данному разделу в норме составляет 126). Для оценки спастичности в паретичной руке применяли шкалу Эшворта [13]. Для оценки мелкой моторики кисти и функциональных движений использовали тест ARAT [14].

Для трехмерного анализа движений пациентов использовали аппаратно-программный комплекс «Видеоанализ-3D Биософт» (Биософт; Россия). Так как движения руки очень разнообразны и вариабельны, для оценки биомеханических параметров была выбрана наименее вариабельная парадигма: «достижение удаленно расположенного объекта» (ричинг). Испытуемые располагались за столом, сидя на стуле без спинки с подлокотниками для обеих рук. Руки располагали на подлокотниках ладонями вниз (кисти лежали на столе). На расстоянии вытянутой руки индивидуально для каждого испытуемого на столе устанавливали стакан с утяжелителем весом 10 г. Испытуемому предлагали дотянуться до стакана, взять его, поднести ко рту, имитируя процесс питья, затем поставить стакан на место и вернуть руку в исходную позицию. При невозможности захватить стакан (грубый парез в кисти) испытуемому предлагали совершить попытку захвата. Для обеспечения максимально автоматизированного движения испытуемым сообщали, что основная цель исследования заключалась в изучении движения, имитирующего питье. Таким образом, движение ричинг выполняли с минимальным акцентом внимания, что позволяло добиться получения 
автоматизированных действий. Измеряли только первую часть движения - доставание удаленно расположенного объекта.

Для изучения внутрисуставных и межсуставных угловых синергий в сагиттальной и фронтальной плоскостях были введены следующие коэффициенты (K) синергий: $\mathrm{K}_{1}$ - отношение объема сгибания в плечевом суставе (ПС) к объему отведения в ПС; $\mathrm{K}_{2}$ - отношение объема разгибания в локтевом суставе (ЛС) к объему сгибания в ПС; $\mathrm{K}_{3}$ - отношение объема разгибания в ЛС к объему отведения в ПС.

В рамках курса реабилитации у пациентов проводили тренировки функционального навыка паретичной руки с применением механотерапевтического экзоскелетного комплекса с разгрузкой веса и обратной связью Armeo Spring (Hocoma; Швейцария), тренировки бимануальных и координационных движений с инструктором-методистом лечебной гимнастики, массаж паретичной руки. Во всех случаях курс реабилитации был успешным.

Статистическую обработку результатов проводили с помощью критериев Манна-Уитни (при сравнении независимых выборок), Уилкоксона (при сравнении зависимых выборок), коэффициента корреляции Спирмена, на персональном компьютере с применением пакета прикладных программ Statsoft Statistica v. 7.0 (StatSoft; США). Данные представляли в виде медианы и 25- и 75\%-х квартилей медианы. Статистически значимыми считали различия при $p<0,05$.

\section{РЕЗУЛЬТАТЫ ИССЛЕДОВАНИЯ}

\section{Клиническая оценка}

При проведении сравнительного анализа данных по шкале Фугл-Мейера у всех пациентов после курса реабилитации наблюдали статистически значимое увеличение активных движений в плече, предплечье, запястье и кисти. Было отмечено также достоверное увеличение объема пассивных движений в локтевом и лучезапястном суставах. Важно заметить, что, согласно шкале Фугл-Мейера, значимо уменьшалась выраженность патологической сгибательной синергии (чем больше балл по шкале ФуглМейера, оценивающий выраженность синергии, тем меньше степень ее выраженности) (табл. 1).

По результатам оценки по шкале Эшворта при проведении статистического анализа было выявлено, что после курса реабилитации значимо снизилась степень спастичности в мышцах сгибателях локтевого сустава ( $p=0,00008)$, мышцах сгибателях запястья ( $p=0,00098)$ и наружных сгибателях пальцев ( $p=0,0022)$. Снижение спастичности в исследуемых группах мышц наблюдали у пациентов как с незначительным и легким (1; $1+)$, так и с более выраженным повышением мышечного тонуса (2).

При анализе клинических данных по шкале Фугл-Мейера нами была выявлена тесная связь степени выраженности патологической сгибательной синергии в руке и степени общего двигательного десицита ( $r=0,81 ; p=0,000000)$. Согласно клинической оценке по шкале Фугл-Мейера, нами были выделены больные с грубым парезом, двигательный десрицит которых составил менее 50\% от максимального балла активных движений (менее 33 баллов), выраженным 50-70\% (34-46 баллов), умеренным - 71-89\% (47-56 баллов) и легким парезом - 90-99\% (57-65 баллов). Для дальнейшего анализа пациенты были разделены на 2 группы: группа 1 - пациенты с легким/умеренным парезом, группа 2 - пациенты с грубым/выраженным парезом.

При сравнительном анализе по отдельным подразделам шкалы Фугл-Мейера оказалось, что достоверное улучшение двигательной функции руки наступало как в проксимальных, так и в дистальных отделах руки в обеих подгруппах (табл. 2).

\section{Видеоанализ движений паретичной руки при выполнении ричинг-теста}

При анализе временных характеристик ричинг-теста было выявлено, что пациентам обеих групп на выполнение целевого движения было необходимо достоверно больше времени, чем здоровому человеку. В случае с грубым/выраженным парезом на выполнение движения достижения удаленно расположенного объекта требовалось статистически достоверно больше времени, чем в норме $(o=0,001)$. Разница во времени между группой здоровых добровольцев и группой с легким/умеренным парезом была менее значительна и составила всего 0,55 с (рис. 1).

При анализе временных характеристик ричинг-теста после курса реабилитации было показано, что у пациентов первой группы (с легким/умеренным парезом руки) происходит статистически значимое уменьшение времени, затрачиваемого на достижение объекта $(p=0,04)$. У пациентов второй группы (грубый/выраженный парез) после курса реабилитации время, затрачиваемое на выполнение

Таблица 1. Медиана показателей (Ме [25\%; 75\%]) двигательных нарушений в руке по подразделам шкалы Фугл-Мейера

\begin{tabular}{|c|c|c|}
\hline \multirow{2}{*}{ Раздел шкалы } & \multicolumn{2}{|c|}{ Группа $(n=25)$} \\
\hline & До лечения & После лечения \\
\hline \multirow{2}{*}{ Общий балл } & \multirow{2}{*}{$103[91 ; 109]$} & $109[99 ; 120]$ \\
\hline & & $p=0,000025$ \\
\hline \multirow{2}{*}{ Движения плеча и предплечья } & \multirow{2}{*}{$29[24 ; 34]$} & $32[24 ; 38]$ \\
\hline & & $p=0,000821$ \\
\hline \multirow{2}{*}{ Движения запястья и кисти } & \multirow{2}{*}{$18[13 ; 21]$} & $20[9 ; 23,5]$ \\
\hline & & $p=0,000168$ \\
\hline \multirow{2}{*}{ Синергии } & \multirow{2}{*}{$9[6 ; 10]$} & $9,5[5 ; 11]$ \\
\hline & & $p=0,000049$ \\
\hline \multirow{2}{*}{ Объем пассивных движений } & \multirow{2}{*}{$21[20 ; 22]$} & $23[22 ; 24]$ \\
\hline & & $p=0,000327$ \\
\hline
\end{tabular}


Таблица 2. Медиана показателей (Ме [25\%; 75\%]) двигательных нарушений в руке по шкале Фугл-Мейера у больных до и после реабилитации

\begin{tabular}{|l|c|c|}
\hline \multicolumn{3}{|c|}{ Движения плеча и предплечья, баллы $(n=25)$} \\
\hline До лечения & Легкий/умеренный парез $(n=13)$ & Грубый/выраженный парез $(n=12)$ \\
\hline После лечения & $34[32 ; 37]$ & $24[21,5 ; 27]$ \\
\hline$p$-level & $38[34 ; 41]$ & $p 0,5[25,5 ; 33,5]$ \\
\hline \multicolumn{2}{|c|}{$p=0,041$} & $p=0068$ \\
\hline \multicolumn{2}{|c|}{ Движения запястья и кисти, баллы $(n=25)$} \\
\hline До лечения & Легкий/умеренный парез $(n=13)$ & Грубый/выраженный парез $(n=12)$ \\
\hline После лечения & $21[19 ; 21]$ & $12[8 ; 14,5]$ \\
\hline$p$-level & $23[22 ; 24]$ & $14[10 ; 19,5]$ \\
\hline
\end{tabular}

этого теста, наоборот, достоверно увеличилось ( $p=0,043)$ и стало превышать соответствующий показатель в норме более чем в 2 раза.

При анализе результатов биомеханического исследования было выявлено, что у больных с легким/умеренным парезом статистически значимо была уменьшена максимальная угловая амплитуда сгибания в плечевом суставе и увеличена максимальная угловая амплитуда отведения в плечевом суставе при выполнении ричингтеста (рис. 2А).

Помимо уменьшения максимального угла движения в некоторых суставах у больных с легким/умеренным парезом увеличивалось время достижения максимумов угловой амплитуды при всех движениях по сравнению с нормой (рис. ЗА, В).

Кинематический портрет в группе больных с грубым/ выраженным парезом был другим: при выполнении движения максимальный угол отведения в плечевом суставе был больше, чем в норме (рис. 2Б), при этом значение максимального угла разгибания в локтевом суставе было значимо ниже нормы (рис. 3Б, В).

Помимо уменьшения максимального угла в некоторых суставах у больных с грубым/выраженным парезом значимо увеличивалось время достижения максимумов угловой амплитуды при всех движениях по сравнению с нормой. У пациентов этой группы обращает на себя внимание изменение времени достижения пиков амплитуд в суставах при движении. Если в группе с легким/умеренным парезом порядок достижения максимальных амплитуд в суставах оставался прежним, то в группе с грубым/выраженным парезом он был иным. Так, отведение плечевого сустава, достигавшее своего пика первым из всех участвующих суставов как в норме, так и при легком/умеренном парезе, у пациентов с грубым/выраженным парезом появлялось только в середине движения, после разгибания в лучезапястном суставе.
При сравнении параметров максимума угловых амплитуд в суставах у пациентов первой группы до и после реабилитации статистически значимых различий в этих показателях обнаружено не было. В то же время при анализе изменения объема движений в суставах после тренировки были получены достоверные изменения биомеханических показателей в плечевом суставе: увеличение объема сгибания ( $p=0,04)$ и уменьшение объема отведения ( $p=0,01)$.

Анализ изменения скоростных параметров движения показал достоверное увеличение угловой скорости сгибания в плечевом суставе $(p=0,01)$, разгибания в локтевом суставе $(p=0,02)$, а также уменьшение угловой скорости отведения в плечевом суставе $(p=0,02)$. При изучении коэффициентов синергий, отражающих межсуставные взаимодействия у пациентов первой группы достоверные различия после курса реабилитации были выявлены только по коэффрициенту $\mathrm{K}_{2}$ ( $\left.p=0,04\right)$, отражающему взаимодействие между сгибанием в плечевом суставе и разгибанием в локтевом суставе во время выполнения ричинг-теста.

При сравнении показателей максимума угловых амплитуд в суставах у пациентов второй группы (грубый) выраженный парез) до и после реабилитации было выявлено значительное уменьшение максимального угла разгибания локтевого сустава $(p=0,01)$. Достоверных изменений в других суставах не было отмечено.

При проведении анализа объема движений в суставах во второй группе пациентов наблюдали изменения, противоположные показателям, полученным у больных первой группы. На фоне проведения реабилитационных мероприятий объем сгибания в плечевом суставе достоверно уменьшался ( $p=0,02)$, при этом также наблюдалось достоверное увеличение объема отведения в плечевом суставе $(p=0,04)$. В локтевом суставе достоверных различий до и после реабилитации обнаружено не было. Так же стоит отметить, что несмотря

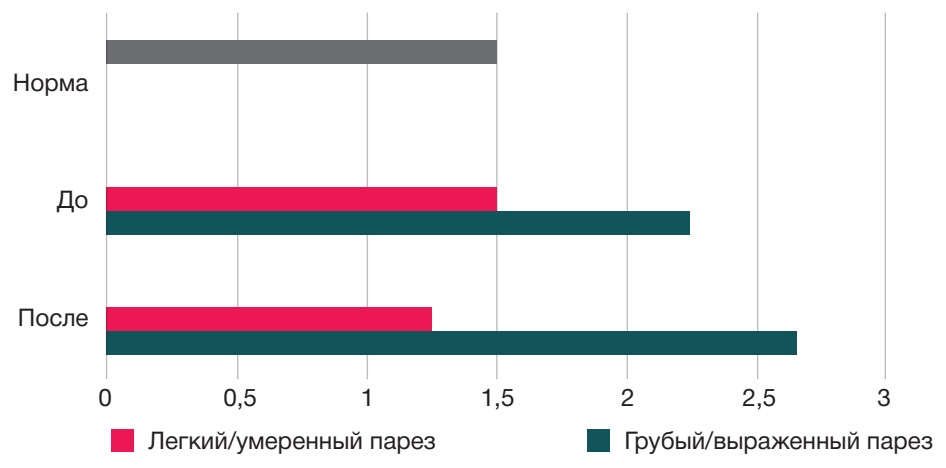

Рис. 1. Время (с) выполнения движения ричинг-теста у больных с разной степенью пареза в руке 
на уменьшение объема сгибания в плечевом суставе, статистически значимых отличий от нормальных значений данного показателя обнаружено не было.

Достоверные отличия объемов движений от нормального двигательного стереотипа сохранялись по остальным показателям: объему отведения в плечевом суставе $(p=0,04)$, объему разгибания в локтевом суставе $(p=0,007)$, объему разгибания в лучезапястном суставе ( $p=0,02)$. Кроме того, были выявлены противоположные изменения в скоростных характеристиках движения у пациентов второй группы по отношению к изменениям у пациентов первой группы. Так, после курса реабилитации отмечали достоверное увеличение угловой скорости отведения в плечевом суставе $(p=0,02)$, в то же время в локтевом суставе произошло значимое уменьшение угловой скорости $(p=0,02)$ при одновременном уменьшении объема разгибания и максимума угловой амплитудь

A

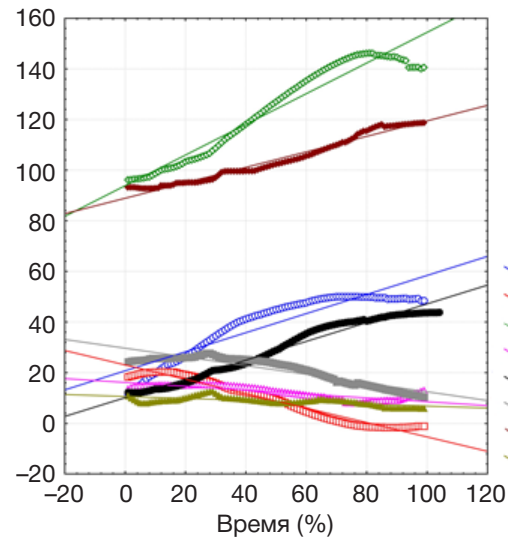

в локтевом суставе. При изучении коэффициентов синергий, отражающих межсуставные взаимодействия, у пациентов второй группы достоверные различия после курса реабилитации, так же как у больных первой группы, отмечали только по коэффициенту $\mathrm{K}_{2}$, который достоверно уменьшался $(p=0,04)$ после курса тренировки.

\section{Анализ биомеханики движений плечевого пояса при выполнении ричинг-теста}

Несмотря на то что полученные результаты показали отсутствие эффекта тренировок на выраженность патологической синергии у больных с грубым/ выраженным парезом в руке, при клинической оценке наблюдали улучшение функциональных возможностей в паретичной руке, что было выражено в достоверном улучшении мелкой моторики по шкале ARAT. В одном

Рис. 2. Межсуставные взаимодействия в паретичной руке у пациентов с легким/умеренным (А) и грубым/выраженным (Б) парезом

A

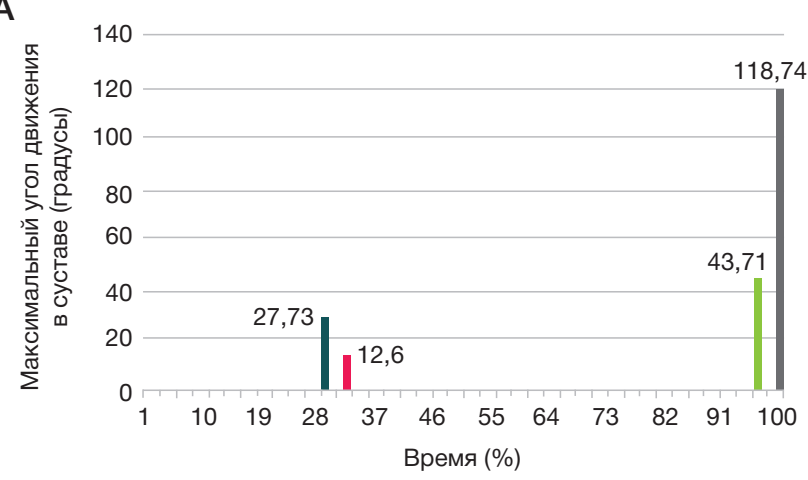

Б

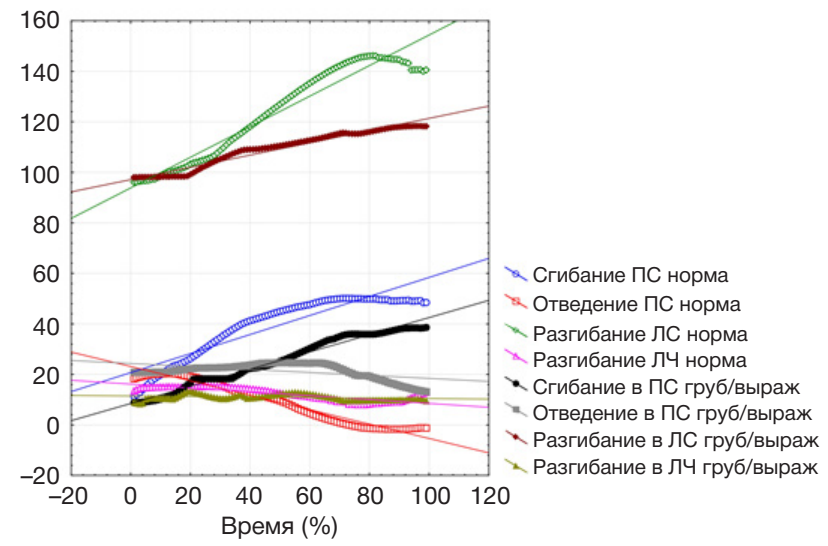

Б

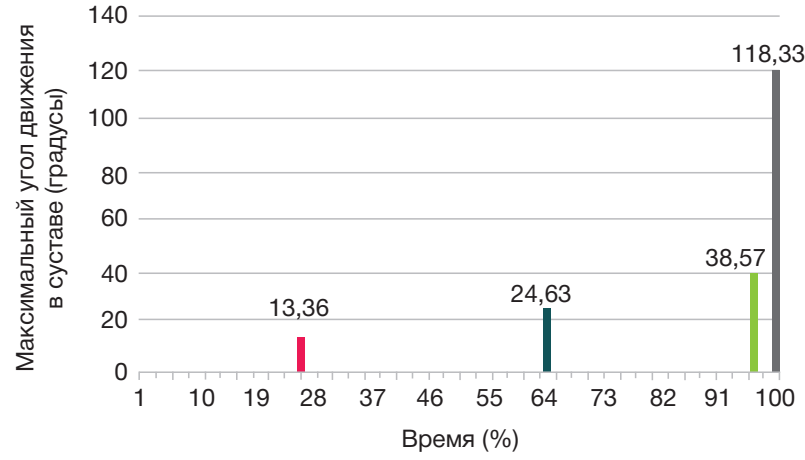

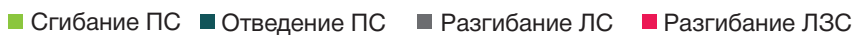

ш Сгибание ПС Отведение ПС $\square$ Разгибание ЛС $\square$ Разгибание ЛзС

B

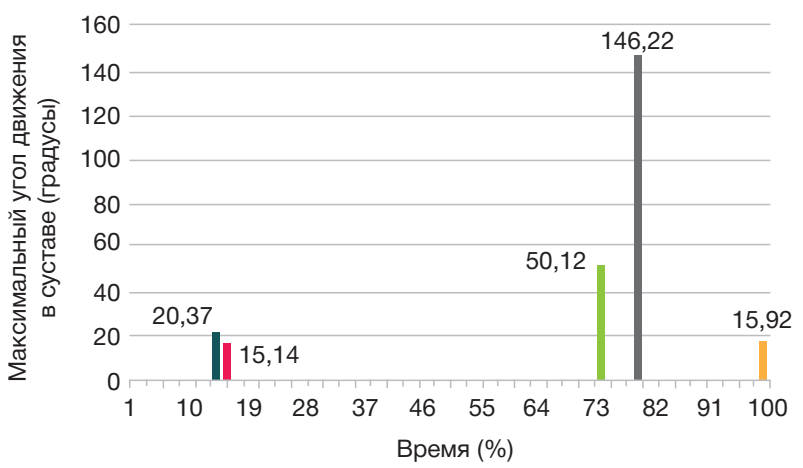

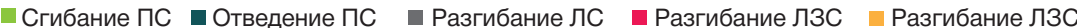

Рис. 3. Максимум угловой амплитуды движения в разных суставах при выполнении ричинг-теста в норме (А) и у больных с легким/умеренным парезом (Б) по сравнению с нормой (B) 
из исследований у пациентов с умеренным парезом с улучшением функциональности по клиническим шкалам наблюдали уменьшение смещения корпуса и плечевого пояса по данным видеоанализа движений $[15,16]$. Для подтверждения гипотезы о наличии компенсаторного движения плечевого пояса у пациентов с грубым/выраженным парезом в руке был проведен дополнительный анализ движений во время выполнения ричинг-теста. Для этой цели оценивали смещение двух маркеров, располагавшихся на акромионе здорового и паретичного плечей во фронтальной плоскости.

Полученные результаты показали смещение плечевого пояса у пациентов с грубым/выраженным парезом в сторону объекта при выполнении ричинг-теста, как до тренировок (23 [19,8; 57,4] - здоровое плечо; 169 [88,0; 178,0] - паретичное плечо), так и после тренировок (66 [49,0; 81,0] - здоровое плечо; 215 [162,0; 229,0] паретичное плечо), с достоверно большим преобладанием смещения паретичного плеча. Помимо этого, проведенный анализ выявил достоверное ( $p=0,04)$ увеличение смещения плечевого пояса вперед при выполнении ричингдвижения на фоне курса реабилитации.

\section{ОБСУЖДЕНИЕ РЕЗУЛЬТАТОВ}

После курса реабилитационного лечения нами были получены данные о том, что обе группы пациентов не только различаются в значительной степени по кинематическому портрету, но и имеют разные пути двигательного восстановления.

Так, у пациентов с легким/умеренным парезом восстановление двигательной функции в паретичной руке происходит по пути нормализации паттерна движения, о чем свидетельствует увеличение коэффициента $\mathrm{K}_{2}$, отражающего межсуставное взаимодействие в плечевом и локтевом суставах, что имеет прямую корреляцию с уменьшением клинической выраженности степени пареза по шкале Фугл-Мейера $(r=0,94 ; p=0,01)$. У пациентов с грубым/выраженным парезом в руке восстановление двигательной функции в паретичной руке происходит по пути компенсации двигательного дефицита, о чем свидетельствует снижение коэффициента $\mathrm{K}_{2}$, что имеет обратную корреляцию с уменьшением клинической выраженности степени пареза по шкале Фугл-Мейера $(r=-0,9 ; p=0,03)$, т. е. у данных пациентов происходит улучшение функциональных движений в руке при сохранении патологического паттерна движения. Дальнейший анализ показал, что у пациентов с грубым/выраженным парезом после курса реабилитации достоверно увеличивалось смещение плечевого пояса вперед при выполнении ричинг-движения. При проведении корреляционного анализа была обнаружена отрицательная взаимосвязь смещения маркера на паретичном плече со значением $\mathrm{K}_{2}(r=-0,9 ; p=0,03)$. Взаимосвязь движений туловища и паретичной конечности подчеркивают и результаты ряда научных исследований [17]. Эти данные свидетельствуют о наличии у пациентов с грубым/выраженным парезом компенсаторного механизма и объясняют снижение этого коэффициента после проведения реабилитации, так как при большем смещении туловища объемы движений и максимальные углы в суставах становились меньше. Можно предположить, что при грубом/выраженном парезе восстановление двигательных навыков идет по пути компенсации, поэтому возвращения к нормальному

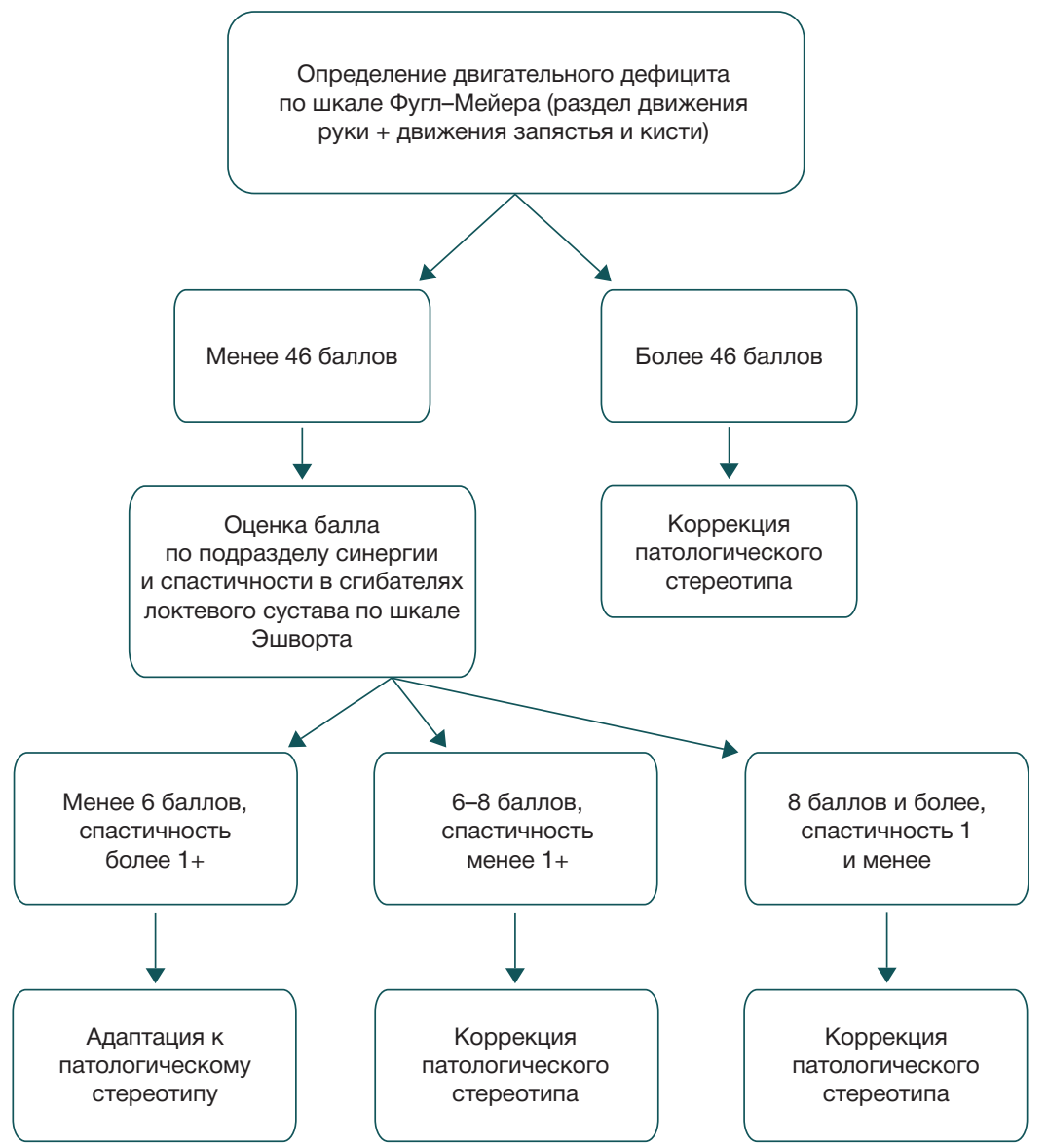

Рис. 4. Алгоритм выбора тактики реабилитационных мероприятий у больных с постинсультным парезом руки 
паттерну движений у пациентов со сформировавшейся в полной степени патологической синергией в руке невозможно. По нашим данным, тренировка, проводимая специалистом по реабилитации, не во всех случаях должна быть направлена на преодоление патологических синергий, поскольку на адаптацию и обучение пациентов с грубым/ выраженным парезом целесообразно использовать максимально эффективно компенсаторные механизмы. Такой вывод нашел подтверждение и при анализе данных клинического осмотра, так как после проведенного курса реабилитации было отмечено достоверное улучшение функциональности паретичной руки в обеих группах пациентов, в частности навыков, связанных с мелкой моторикой. Мы предполагаем, и это сопоставимо с данными многих мировых исследований [18-21], что этот эффект может быть связан с отсутствием ограничения степеней свободы в паретичной конечности во время тренировки, так как пациенты обучались действовать в рамках своего стереотипа и преодолевать его при необходимости произвольно.

На основании полученных клинико-биомеханических данных в группах пациентов с разной степенью спастичности и выраженностью пареза в руке был разработан алгоритм выбора тактики реабилитационных мероприятий у больных с постинсультным парезом руки (рис. 4). При этом оценку до начала курса реабилитации и разработки реабилитационной стратегии необходимо проводить по подразделу шкалы Фугл-Мейера для верхней конечности. Стоит отметить, что оценка по шкале Эшворта также необходима и обязательно должна быть проведена в трех мышечных группах: сгибателях локтевого и лучезапястного суставов и сгибателях пальцев. Степень спастичности, влияющая на выбор тактики ведения пациента, составляет 1+ в двух и более мышечных группах.

\section{ВЫВОДЫ}

Проведенное детальное клинико-биомеханическое исследование динамики изменений кинематического портрета одного из самых функционально значимых для человека движений (ричинг-теста) на фоне реабилитационных мероприятий показало, что определяющее значение для наиболее эффективного и успешного восстановления функции паретичной руки имеют исходная тяжесть поражения и степень спастичности. Именно они определяют формирование патологических двигательных синергий при постинсультном парезе руки и обусловливают включение различных механизмов трансформации двигательного стереотипа в процессе восстановления. Полученные данные позволили сформировать алгоритм выбора тактики реабилитационных мероприятий, основанный прежде всего на клинических показателях: у больных с легким/умеренным парезом целесообразно проводить тренировку в рамках физиологического паттерна движений с подавлением компенсаторных механизмов, направленную на коррекцию патологического стереотипа; у больных с грубым/выраженным парезом в руке, напротив, необходимы тренировки с поощрением механизмов компенсации и повышением функциональности паретичной руки в рамках сформировавшегося патологического стереотипа.

\section{Литература}

1. Lawrence ES, Coshall C, Dundas R, et al. Estimates of the prevalence of acute stroke impairments and disability in a multiethnic population. J Stroke. 2001; (32): 1279-84.

2. Persson HC, Parziali M, Danielsson A, Sunnerhagen KS. Outcome and upper extremity function within 72 hours after first occasion of stroke in an unselected population at a stroke unit. A part of the SALGOT study. J BMC Neurol. 2012; (12): 162.

3. Langhorne P, Coupar F, Pollock A. Motor recovery after stroke: a systematic review. Lancet Neurol. 2009; 8 (8): 741-54.

4. Veerbeek JM, Kwakkel G, van Wegen EE, Ket JC, Heymans MW Early prediction of outcome of activities of daily living after stroke: a systematic review. Stroke. 2011; 42 (5): 1482-8.

5. Brunnstrom S. Movement Therapy in Hemiplegia: A Neurophysiological Approach. Facts and Comparisons. NewYork: Harper and Row, 1970.

6. Santello M, Lang CE. Are movement disorders and sensorimotor injuries pathologic synergies? When normal multi-joint movement synergies become pathologic. J Front Hum Neurosci. 2015; (8): 1050.

7. van Kordelaar J, van Wegen EE, Kwakkel G. Unraveling the interaction between pathological upper limb synergies and compensatory trunk movements during reach-to-grasp after stroke: a cross-sectional study. J Exp Brain Res. 2012; 221 (3): 251-62.

8. Van Vliet PM, Sheridan MR. Coordination between reaching and grasping in patients with hemiparesis and healthy subjects. J Arch Phys Med Rehabil. 2007; (88): 1325-31.

9. Hogan L, Dipietro HI, Krebs SE, et al. Changing Motor Synergies in Chronic Stroke. J Neurophysiol. 2007; (98): 757-68.

10. Compston $\mathrm{A}$. Aids to the investigation of peripheral nerve injuries. Medical Research Council: Nerve Injuries Research Committee. His Majesty's Stationery Office: 1942; pp. 48 (iii) and 74 figures and 7 diagrams; with aids to the examination of the peripheral nervous system. By Michael O'Brien for the Guarantors of Brain. Saunders Elsevier. Brain. 2010; 133 (10): 2838-44.

11. Oldfield RC. The assessment and analysis of handedness: the Edinburgh inventory. Neuropsychologia. 1971; (9): 97-113.

12. Sanford J, Moreland J, Swanson LR, Stratford PW. Reliability of the Fugl-Meyer assessment for testing motor performance in patients following stroke. J Gowland C Phys Ther. 1993; 73 (7): $447-54$

13. Bohannon RW, Smith MB. Interrater reliability of a modified Ashworth scale of muscle spasticity. J Phys Ther. 1987; 67 (2): 206-7.

14. Doussoulin SA, Rivas SR, Campos SV. Validation of «Action Research Arm Test» (ARAT) in Chilean patients with a paretic upper limb after a stroke. Rev Med Chil. 2012; 140 (1): 59-65.

15. Alt Murphy M, Willén C, Sunnerhagen KS. Movement kinematics during a drinking task are associated with the activity capacity level after stroke. J Neurorehabil Neural Repair. 2012; 26 (9): 1106-15.

16. Valdés BA, Glegg SMN, Van der Loos HFM. Trunk Compensation During Bimanual Reaching at Different Heights by Healthy and Hemiparetic Adults. J Mot Behav. 2017; 49 (5): 580-92.

17. van Kordelaar J, van Wegen EE, Kwakkel G. Unraveling the interaction between pathological upper limb synergies and compensatory trunk movements during reach-to-grasp after stroke: a cross-sectional study. J Exp Brain Res. 2012; 221 (3): 251-62.

18. Roh J, Rymer WZ, Perreault EJ, et al. Saturated muscle activation contributes to compensatory reaching strategies after stroke. $J$ Neurophysiol. 2013; 109 (3): 768-81.

19. Basteris A, Nijenhuis SM, Stienen AH, et al. Training modalities in robot-mediated upper limb rehabilitation in stroke: a framework 
for classification based on a systematic review. J Neuroeng Rehabil. 2014; 10 (11): 111.

20. Daunoraviciene K, Adomaviciene A, Grigonyte A, Griškevičius J, Juocevicius A. Effects of robot-assisted training on upper limb functional recovery during the rehabilitation of poststroke patients J Technol Health Care. 2018; 26 (2): 533-42.

\section{References}

1. Lawrence ES, Coshall C, Dundas R, et al. Estimates of the prevalence of acute stroke impairments and disability in a multiethnic population. J Stroke. 2001; (32): 1279-84.

2. Persson HC, Parziali M, Danielsson A, Sunnerhagen KS. Outcome and upper extremity function within 72 hours after first occasion of stroke in an unselected population at a stroke unit. A part of the SALGOT study. J BMC Neurol. 2012; (12): 162.

3. Langhorne P, Coupar F, Pollock A. Motor recovery after stroke: a systematic review. Lancet Neurol. 2009; 8 (8): 741-54.

4. Veerbeek JM, Kwakkel G, van Wegen EE, Ket JC, Heymans MW Early prediction of outcome of activities of daily living after stroke: a systematic review. Stroke. 2011; 42 (5): 1482-8.

5. Brunnstrom S. Movement Therapy in Hemiplegia: A Neurophysiological Approach. Facts and Comparisons. NewYork: Harper and Row, 1970.

6. Santello M, Lang CE. Are movement disorders and sensorimotor injuries pathologic synergies? When normal multi-joint movement synergies become pathologic. J Front Hum Neurosci. 2015; (8): 1050.

7. van Kordelaar J, van Wegen EE, Kwakkel G. Unraveling the interaction between pathological upper limb synergies and compensatory trunk movements during reach-to-grasp after stroke: a cross-sectional study. J Exp Brain Res. 2012; 221 (3): 251-62.

8. Van Vliet PM, Sheridan MR. Coordination between reaching and grasping in patients with hemiparesis and healthy subjects. J Arch Phys Med Rehabil. 2007; (88): 1325-31.

9. Hogan L, Dipietro HI, Krebs SE, et al. Changing Motor Synergies in Chronic Stroke. J Neurophysiol. 2007; (98): 757-68.

10. Compston A. Aids to the investigation of peripheral nerve injuries. Medical Research Council: Nerve Injuries Research Committee. His Majesty's Stationery Office: 1942; pp. 48 (iii) and 74 figures and 7 diagrams; with aids to the examination of the peripheral nervous system. By Michael O'Brien for the Guarantors of Brain. Saunders Elsevier. Brain. 2010; 133 (10): 2838-44.

11. Oldfield RC. The assessment and analysis of handedness: the Edinburgh inventory. Neuropsychologia. 1971; (9): 97-113.
21. Устинова К. И., Черникова Л. А., Хижникова А. Е., Пойдашева А. Г., Супонева Н. А., Пирадов М. А. Теоретическое обоснование классических методов двигательной реабилитации в неврологии. Анналы клинической и экспериментальной неврологии. 2018; 12 (3): 54-60.

12. Sanford J, Moreland J, Swanson LR, Stratford PW. Reliability of the Fugl-Meyer assessment for testing motor performance in patients following stroke. J Gowland C Phys Ther. 1993; 73 (7): 447-54.

13. Bohannon RW, Smith MB. Interrater reliability of a modified Ashworth scale of muscle spasticity. J Phys Ther. 1987; 67 (2): 206-7.

14. Doussoulin SA, Rivas SR, Campos SV. Validation of «Action Research Arm Test» (ARAT) in Chilean patients with a paretic upper limb after a stroke. Rev Med Chil. 2012; 140 (1): 59-65.

15. Alt Murphy M, Willén C, Sunnerhagen KS. Movement kinematics during a drinking task are associated with the activity capacity level after stroke. J Neurorehabil Neural Repair. 2012; 26 (9): 1106-15.

16. Valdés BA, Glegg SMN, Van der Loos HFM. Trunk Compensation During Bimanual Reaching at Different Heights by Healthy and Hemiparetic Adults. J Mot Behav. 2017; 49 (5): 580-92.

17. van Kordelaar J, van Wegen EE, Kwakkel G. Unraveling the interaction between pathological upper limb synergies and compensatory trunk movements during reach-to-grasp after stroke: a cross-sectional study. J Exp Brain Res. 2012; 221 (3): 251-62.

18. Roh J, Rymer WZ, Perreault EJ, et al. Saturated muscle activation contributes to compensatory reaching strategies after stroke. J Neurophysiol. 2013; 109 (3): 768-81.

19. Basteris A, Nijenhuis SM, Stienen AH, et al. Training modalities in robot-mediated upper limb rehabilitation in stroke: a framework for classification based on a systematic review. J Neuroeng Rehabil. 2014; 10 (11): 111.

20. Daunoraviciene K, Adomaviciene A, Grigonyte A, Griškevičius J, Juocevicius A. Effects of robot-assisted training on upper limb functional recovery during the rehabilitation of poststroke patients. J Technol Health Care. 2018; 26 (2): 533-42.

21. Ustinova KI, Chernikova LA, Khizhnikova AE, Poydasheva AG, Suponeva NA, Piradov MA. Theoretical basis for classical methods of motor rehabilitation in neurology. Annals of clinical and experimental neurology. 2018; 12 (3): 54-60. 